\title{
Valoración de un Programa de Entrenamiento en Estilo Explicativo Optimista y Habilidades de Comunicación en Adolescentes
}

\section{Evaluation of a Training Program to promote Optimistic Explanatory Style and Communication Skills in adolescents}

\author{
Juan Molina Hernández \\ Ana García León ${ }^{1}$ \\ Universidad de Jaén, España \\ (Rec: julio de 2015 - Acept: noviembre de 2015)
}

\begin{abstract}
Resumen
El estilo explicativo pesimista y las dificultades de interacción social han sido asociados con un incremento de los síntomas depresivos durante la adolescencia. Para disminuir esta sintomatología, se han propuesto algunos programas de entrenamiento centrados en la modificación de uno o ambos elementos. En este estudio se pretende valorar la eficacia de un programa de entrenamiento para fomentar el estilo explicativo optimista y mejorar las habilidades comunicativas. Los participantes han sido 38 adolescentes (19 chicos y 19 chicas), con edades entre 14 y 17 años $(M=14.93$; D.T. = 0.87). La mitad fue asignada al azar a un grupo de tratamiento y la otra mitad a un grupo de control de lista de espera. El programa produjo una disminución en los síntomas depresivos $(\mathrm{p}<.001)$, así como una mejora en la satisfacción con la vida $(\mathrm{p}<.001)$ y las habilidades de interacción con otros adolescentes $(\mathrm{p}<.01)$. También se detecta el aumento del tamaño del efecto entre el grupo tratamiento y el grupo control, a favor del primero. Los resultados confirman el beneficio de aplicar programas de entrenamiento centrados en estos componentes para reducir los síntomas depresivos en los adolescentes.
\end{abstract}

Palabras clave: adolescentes, estilo explicativo optimista/pesimista, programas de entrenamiento, satisfacción vital, síntomas depresivos.

\begin{abstract}
Pessimistic explanatory style and difficulties in social interaction have been associated with an increase of depressive symptoms during adolescence. Some training programs, focused on the modification of one or both factors, have been proposed to decrease such symptoms. This study examines the efficacy of a training program focused on both optimistic explanatory style and communication skills for this population. Thirty-eight adolescents (19 males an 19 females) aged between 14 and $17(\mathrm{M}=14.93$; D.T. $=0.87)$ were randomly assigned to a treatment group (50\%) or a control group (50\%). The intervention program produced both a decrease of depressive symptoms $(\mathrm{p}<.001)$, and an increase of satisfaction with life $(\mathrm{p}<.001)$ and the interaction skills with other adolescents $(\mathrm{p}<.01)$. In addition, effects size differences between both groups (treatment and control group) increased at post-treatment compared to pre-treatment. In conclusion, this training program shows to be effective in reducing depressive symptoms in adolescents.
\end{abstract}

Keywords: adolescents, depressive symptoms, optimistic/pessimistic explanatory style, life satisfaction, training programs.

\footnotetext{
1 Correspondencia a: Ana García León. Departamento de Psicología. Universidad de Jaén. Campus de las Lagunillas, S/N, 23071 Jaén, España. E-mail: anagarcia@ujaen.es
} 


\section{Introducción}

La adolescencia es una etapa de grandes cambios de distinta naturaleza (físicos, sexuales, cognitivos, sociales...) que se producen en muy poco tiempo (Berger, 1993; Frydenberger, 1997). Estos cambios, y la adaptación a ellos, aumentan la vulnerabilidad de los adolescentes y pueden favorecer el desarrollo de problemas emocionales y de conducta. De hecho, los síntomas depresivos y la depresión clínica se incrementan durante la adolescencia (Chaplin et al., 2006; Griciute, 2011; Hankin, 2008; Nrughma, Holen \& Sund, 2010), especialmente entre las chicas (Hankin \& Abramson, 2001; Twenge \& Nolen-Hoeksema, 2002), siendo muy frecuente experimentar el primer episodio de depresión durante este período (Southwick, Litz, Charney \& Friedman, 2011). Además, la depresión clínica suele estar asociada durante estos momentos con otros problemas como consumo de drogas, rechazo y aislamiento social, problemas académicos y suicidio (Chaplin et al., 2006; Gillham \& Reivich, 2004).

Algunos/as investigadores/as consideran que el tipo de cognición que se produce durante la depresión emerge durante la adolescencia: cuando los niños y niñas empiezan a desarrollar un sentido de la identidad personal más estable y menos concreto, así como la capacidad para realizar atribuciones sobre los acontecimientos (Garber, 2000). El estilo atribucional hace referencia al modo en que las personas explican las causas de los acontecimientos positivos y negativos, una vez que éstos han sucedido. Así, se considera que las personas que atribuyen los acontecimientos positivos a factores internos, estables y globales, y los acontecimientos negativos a factores externos, inestables y específicos, tendrían un estilo atribucional optimista. Por su parte, las personas con un estilo explicativo pesimista son aquellas que atribuyen los acontecimientos positivos a factores externos, inestables y específicos y los eventos negativos a factores internos, estables y globales (Gillham, Shatte, Reivich $\&$ Seligman, 2001). Se ha sugerido que las expectativas sobre el futuro son afectadas por el estilo explicativo; de este modo, una explicación pesimista de los eventos puede disminuir la motivación, reducir la persistencia y hacer a las personas más susceptibles a la depresión. Numerosos estudios han mostrado que los adultos con un estilo explicativo pesimista presentan más síntomas depresivos cuando se enfrentan con situaciones problemáticas (Alloy et al., 2006; Chan, 2012; Know \& Laurenceau, 2002; Mongrain \& Blackburn, 2005; Sturman, Mongrain \& Kohn, 2006). En el caso concreto de la adolescencia, otras investigaciones realizadas asocian también el estilo explicativo pesimista con un incremento en el riesgo para experimentar síntomas depresivos (Gibb \& Alloy, 2006; Gillham, Brunwasser \& Freres, 2008; Jacobs, Reinecke, Gollan \& Kane, 2008). Por último, la falta de destrezas interpersonales de los adolescentes también se ha relacionado positivamente con los síntomas depresivos (Rodríguez, Donnenberg, Emerson, Wilson \& Javdani, 2015).

En las tres últimas décadas se han llevado a cabo varios programas de entrenamiento que pretenden la modificación del estilo explicativo pesimista como modo de disminuir los síntomas depresivos en niños y adolescentes. El Programa de Optimismo de Pennsylvania (POP) fue el primer estudio longitudinal diseñado con este objetivo (Nolen-Hoeksema, Girgus \& Seligman, 1992; Seligman et al., 1984). Este programa se llevó a cabo de modo grupal con niños y niñas de primaria (8-12 años) y constó de dos componentes básicos: uno cognitivo (para enseñar a detectar las atribuciones y predicciones pesimistas y ayudarles a pensar de modo más adecuado) y uno interpersonal (para enseñar estrategias y habilidades útiles para mejorar su relación con los demás). Aunque el estudio presentaba algunos problemas metodológicos, el entrenamiento generó un estilo explicativo más optimista para los acontecimientos negativos y redujo los síntomas depresivos, manteniéndose estos resultados durante el período de seguimiento de dos años (Gillham, Reivich, Jaycox \& Seligman, 1995; Shatté, Gillham \& Reivich, 2000). Un estudio de replicación de este programa, realizado en Estados Unidos con niños y niñas latinas y de raza negra de 11 y 12 años, demostró que el programa reducía sólo los síntomas depresivos de los niños y niñas latinas y no cambiaba las atribuciones en ninguno de los dos grupos (Cardemil, Reivich \& Seligman, 2002). Otro estudio más reciente, realizado con chicos y chicas con edades entre 10 y 12 años, y en el que se ha usado el componente cognitivo del POP, mostró la existencia de mayores niveles de satisfacción con la vida en adolescentes a los que se había aplicado el programa, en comparación con un grupo de control (Suldo, Savage \& Mercer, 2014).

Otro programa de prevención planteado en la misma línea ha sido el Programa de Resiliencia de Pennsylvania (PRP; Gillham et al., 2008; Lewinsohn, Pettit, Joiner \& Seeley, 2003). Se ha desarrollado con niños/as y adolescentes de edades comprendidas entre los 10 y los 14 años y su objetivo era enseñar a los participantes la conexión entre los acontecimientos que les suceden, sus creencias sobre ellos y las consecuencias 
emocionales de sus interpretaciones. En comparación con un grupo de control, el programa fue eficaz para reducir los síntomas depresivos en ambos sexos y tuvo efectos positivos sobre el estilo cognitivo de los/ as niños/as, manteniéndose estos efectos durante el seguimiento de un año (Brunwasser, Gillham \& Kim, 2009; Jaycox, Reivich, Gillham \& Seligman, 1994). Recientemente, se han examinado los efectos de este programa para prevenir los episodios clínicos de depresión en adolescentes, con edades entre 11 y 13 años, y con alto riesgo para su desarrollo (Gillham, Hamilton, Freres, Patton \& Gallop, 2006), encontrándose que sólo se mejoraba el estilo explicativo para los eventos positivos y se reducían los síntomas depresivos en las chicas. El PRP ha demostrado igualmente su eficacia con versiones del mismo aplicadas en otros países. En Holanda, en concreto, se ha adaptado este programa con éxito para prevenir los síntomas depresivos en adolescentes con edades entre 11 y 15 años, así como para disminuir los síntomas depresivos en chicas adolescentes que ya los muestran (Kindt, Van Zundert \& Engels, 2012; Tak et al., 2012). En este último caso, las chicas que realizaron las sesiones del programa mostraban niveles más bajos de sintomatología depresiva que las adolescentes del grupo de control, encontrándose estos efectos incluso 6 meses después de concluido el entrenamiento (Wijnhoven, Creemer, Vermulst, Scholte $\&$ Engels, 2014). Por su parte, en Macedonia, una adaptación de este programa con adolescentes entre 14-16 años, fue capaz de desarrollar un estilo explicativo más optimista y reducir la depresión en los adolescentes del estudio (Miloseva, 2012).

También en la última década, se han elaborado y aplicado otros programas de intervención para fomentar el estilo explicativo optimista, la esperanza, la resiliencia y el bienestar, así como para disminuir los síntomas depresivos. Uno de ellos es el Programa para fomentar la Resiliencia y las Actitudes Optimistas en los Estudiantes (SOAR; Gilboy, 2003), realizado con adolescentes entre 11 y 14 años, cuyos objetivos consistían en que los estudiantes adquirieran destrezas para combatir los pensamientos negativos y aumentaran su flexibilidad cognitiva y su resistencia futura al fracaso y a este tipo de pensamientos. Los resultados mostraron que los/as estudiantes que recibieron un entrenamiento en este programa mostraban niveles menores de depresión que los estudiantes del grupo de control, siendo estos resultados más marcados en aquellos/as estudiantes que estaban más deprimidos inicialmente. Sin embargo, estos cambios en los síntomas depresivos no se mantenían 5 meses después de concluido el tratamiento, ni tampoco se encontraron diferencias entre los grupos en el desarrollo de un estilo explicativo más optimista, corroborando, esto último, los resultados de otros estudios anteriores (Cardemil et al., 2002). Por último, Cantrell (2006) ha comparado la eficacia de un entrenamiento para disminuir los pensamientos pesimistas con respecto a los acontecimientos negativos con uno dirigido a disminuir los pensamientos pesimistas que se producen ante los eventos positivos. Ambos tratamientos demostraron ser efectivos para reducir los niveles de depresión e incrementar la felicidad en los/as adolescentes (con edades entre 16 y 18 años); no obstante, parece que centrar el entrenamiento en los acontecimientos positivos produce mayores incrementos de los niveles de felicidad y de placer al experimentar los eventos cotidianos.

Como puede comprobarse mediante la lectura de los párrafos anteriores, son varios los programas que se han diseñado y aplicado en distintos países para disminuir los síntomas depresivos de los adolescentes, fomentando un estilo explicativo más optimista. En España, aunque existen algunos estudios que relacionan el estilo explicativo pesimista con una mayor sintomatología depresiva en adultos y adolescentes (Sánchez Hernández \& Méndez Carrillo, 2009; Sanjuán \& Magallares, 2006, 2009; Sanjuán, Pérez-García, Rueda \& Ruiz, 2008), no se han difundido datos sobre la aplicación de programas de entrenamiento que evalúen los efectos que sobre los síntomas depresivos de los/as adolescentes tiene el desarrollo de un estilo explicativo optimista. El presente estudio aborda esta línea.

El objetivo principal del estudio fue determinar la eficacia de un programa de entrenamiento para adolescentes, mediante el que se pretendía fomentar el estilo explicativo optimista y mejorar las habilidades comunicativas. En primer lugar, se esperaba que el programa produjera un cambio hacia un estilo explicativo más optimista en el grupo de adolescentes a los que se les aplicó, en comparación con el grupo de control. En segundo lugar, se esperaba que el programa de entrenamiento redujera los síntomas depresivos y aumentara las habilidades de interacción y la satisfacción con la vida en los adolescentes del grupo experimental, no sucediendo así en el grupo de control, por lo que las diferencias entre ambos grupos debían ser estadísticamente significativas en estas variables. En tercer lugar, se esperaba que los tamaños del efecto mostrasen cambios en la dirección señalada. Por último, se pretendía estimar si existen diferencias en los efectos del programa relacionados con el sexo de los participantes. 


\section{Metodología}

\section{Participantes}

Se aplicó el Inventario de Depresión Infantil de Kovack (CDI; 1992) a un total de 144 alumnos/as de $3^{\circ}$ de Educación Secundaria Obligatoria (E.S.O.), con edades comprendidas entre 14 y 17 años $(\mathrm{M}=14.93$; D.T. $=0.87)$. Este grupo constituía la totalidad de alumnos/as que estudiaban en tres centros públicos de enseñanza secundaria de la provincia de Jaén, que fueron seleccionados por pertenecer a distintos puntos geográficos de esta provincia. De acuerdo con el criterio establecido por Kovack (1992) para muestras homogéneas o cuando el objetivo es el establecimiento de una línea base para la implantación de un tratamiento (ver descripción del CDI en el apartado de instrumentos para ampliar esta información), se tomó como punto de corte para la inclusión en el estudio haber obtenido una puntuación directa de 13 puntos o más en dicho instrumento. Fueron 38 los/as adolescentes que cumplieron tal criterio: 19 chicos y 19 chicas, cuyas edades estaban comprendidas entre 14 y 17 años $(M=14.93$; D.T. = 0.87). Diecinueve de ellos/as fueron asignados al azar al grupo experimental y los 19 restantes fueron asignados del mismo modo al grupo control. No había diferencias significativas entre ambos grupos en edad, sexo ni nivel de sintomatología depresiva.

El grupo experimental estaba constituido por 8 chicas y 11 chicos, con edades comprendidas entre 14 y 17 años $(\mathrm{M}=14.88$; D.T. $=0.96)$. Recibió un entrenamiento en técnicas para fomentar el pensamiento positivo y mejorar las habilidades de comunicación. El grupo control estaba formado por 11 chicas y 8 chicos, con edades comprendidas entre 14 y 16 años $(M=15$; D.T. $=0.78)$. No recibió ningún entrenamiento en principio, pasando a constituir un grupo de lista de espera. Cuando concluyó la recogida de datos correspondiente al seguimiento, recibió el mismo entrenamiento que se había proporcionado al grupo experimental.

Ninguno de los participantes del estudio abandonó el mismo antes de la finalización del seguimiento.

\section{Consideraciones éticas}

Todos los alumnos participaron voluntariamente en el estudio y se contó con el consentimiento de los adolescentes, de los consejos escolares y de los padres y madres de los participantes.

\section{Instrumentos}

Se han utilizado los siguientes autoinformes:

1. Cuestionario Revisado de Estilo Atribucional para Niños (CASQ-T; Thompson, Kaslow, Weiss \& Nolen-Hoeksema, 1998), en la versión española de López Rodríguez (2003). Consta de 48 ítems que pretenden evaluar el estilo explicativo o atributivo frente a distintos tipos de acontecimientos. Los elementos que lo componen se distribuyen en las seis siguientes subescalas: 1) estilo explicativo para los acontecimientos negativos a partir de la dimensión temporal-permanente, 2) estilo atributivo para los acontecimientos positivos a partir de la dimensión temporal-permanente, 3) estilo explicativo para los acontecimientos negativos a partir de la dimensión global-específica, 4) estilo atributivo para los acontecimientos positivos a partir de la dimensión global-específica, 5) estilo explicativo para los acontecimientos negativos a partir de la dimensión interna-externa y 6) estilo atributivo para los acontecimientos positivos a partir de la dimensión interna-externa. A partir de estas subescalas, pueden obtenerse tres puntuaciones compuestas: una para los acontecimientos negativos (suma de las tres subescalas para los eventos negativos), otra para los acontecimientos positivos (suma de las tres subescalas para los eventos positivos) y una total (diferencia entre la puntuación para los acontecimientos positivos y la puntuación para los acontecimientos negativos). De estas tres puntuaciones compuestas, se usó en nuestro estudio sólo la escala total. De acuerdo con los primeros datos psicométricos aportados, la consistencia interna para las distintas subescalas osciló entre 0.44 y 0.75 . Por su parte, la estabilidad temporal variaba entre 0.57 y 0.70 . En general, puede afirmarse que los coeficientes de consistencia interna encontrados con respecto a esta prueba son moderados (Schwartz, Kaslow, Seeley \& Lewinsohn, 2000; Seligman et al., 1984); no obstante, la fiabilidad test-retest parece mejorar a medida que aumenta la edad (Nolen-Hoeksema et al., 1992). En la validación española de López Rodríguez (2003), se confirma la estructura factorial de las puntuaciones compuestas para los acontecimientos positivos y los acontecimientos negativos, estando sus índices de consistencia interna entre 0.85 y 0.90 .

2. Inventario de Depresión Infantil (CDI) de Kovacs (1992), en la versión española de Del Barrio y 
Carrasco (2004). Consta de 27 ítems con tres alternativas de respuesta, en función del grado o intensidad de la sintomatología depresiva. Evalúa los síntomas cognoscitivos, emocionales y psicosomáticos, los problemas escolares y sociales y otras conductas. Con muestras amplias de sujetos suele establecerse una puntuación directa de 19 como punto de corte para el diagnóstico de depresión; sin embargo, cuando la muestra es homogénea y lo que interesa es el punto de vista clínico de cara a la implantación de un tratamiento, el punto de corte suele situarse en las puntuaciones directas de $12 \mathrm{o}$ 13 (Kovacs, 1992). La consistencia interna del instrumento ha sido estudiada desde el comienzo de su uso por distintos investigadores, en diferentes países, mostrando la mayoría de los estudios realizados que el instrumento es fiable (en la mayor parte de ellos se han encontrado coeficientes alfa que oscilan entre 0.71 y 0.94$)$; por ejemplo, en la adaptación española de Del Barrio y Carrasco (2004) se obtiene un alfa de Cronbach de 0.79 , una fiabilidad test-retest de 0.38 y una fiabilidad de las dos mitades de 0.77.

3. Cuestionario de Evaluación de Dificultades Interpersonales en la Adolescencia (CEDIA) de Inglés Saura, Méndez e Hidalgo (2000). Este cuestionario consta de 36 ítems con 5 opciones de respuesta y permite identificar a los adolescentes con dificultades en sus relaciones, independientemente de que su origen sea por un déficit en habilidades sociales, debido a la existencia de distorsiones cognitivas y/o por emociones negativas (ansiedad, ira, etc.). Consta de cinco subescalas: 1) asertividad, 2) relaciones heterosexuales, 3) hablar en público, 4) relaciones familiares y 5) relaciones con los amigos. Respecto a la consistencia interna, Inglés Saura et al. (2000) han encontrado valores alfa entre 0.91 y 0.57 . Por su parte, en cuanto a la fiabilidad test-retest, los valores hallados oscilan entre 0.79 y 0.43 (Inglés, Méndez, Hidalgo, Rosa \& Orgilés, 2003). De acuerdo con los objetivos planteados, en este estudio se utilizaron únicamente los datos de las subescalas de relaciones heterosexuales y relaciones con los amigos, cuyos índices de consistencia interna son de 0.85 y de 0.57 , respectivamente. En este último caso, aunque la consistencia interna es moderada, puede considerarse un índice apropiado al constar de pocos elementos.

4. Escala de Satisfacción con la Vida (ESV) de Diener, Emmons, Larsen y Griffin (1985). Esta escala mide el grado de satisfacción general de una persona con su vida. Es una escala unidimensional de cinco ítems formulados de modo positivo, que se responden con una escala Likert de cinco puntos. Diener et al. (1985) encontraron un valor de 0.82 para la fiabilidad test-retest del instrumento, así como un valor de 0.87 para la consistencia interna.

\section{Diseño}

Se utilizó un diseño experimental con un factor intra (pretratamiento, postratamiento y seguimiento) y dos factores inter: tratamiento (grupo de tratamiento versus grupo de control de lista de espera) y género (varón o mujer).

\section{Procedimiento}

El estudio ha constado de cuatro fases: 1) evaluación pretratamiento, 2) aplicación de las técnicas para fomentar el pensamiento positivo y evaluación, 3) aplicación de las técnicas para mejorar las habilidades de comunicación y evaluación y 4) seguimiento de los efectos del tratamiento y evaluación. Todas las pruebas de evaluación aplicadas en el estudio fueron contrabalanceadas en cada una de estas fases.

En la fase de evaluación pretratamiento se administraron el CEDIA, el CASQ-T y la ESV a todos los participantes del estudio. La aplicación fue realizada de forma colectiva en un aula asignada y en horario de tutorías. Previamente, todos los y las participantes habían rellenado el CDI. En la segunda fase, se entrenó al grupo experimental de acuerdo con lo descrito en los componentes Educativo y de Entrenamiento en Optimismo. Se realizó en las mismas condiciones que la primera fase, aunque el grupo de 19 participantes se dividió al azar en tres subgrupos de 6, 6 y 7 personas. Se consideró que era realmente difícil poder aplicar estos componentes con una participación activa de todos y cada uno de los participantes si no se hacían subgrupos; estos subgrupos debían ser necesariamente de entre 5-8 participantes, para que hubiera interacciones sociales suficientes y se pudiese aprender de las situaciones, pensamientos y consecuencias de otras personas, pero sin que el número de participantes coartase las interacciones. Como el $\mathrm{n}^{\mathrm{o}}$ de adolescentes del grupo experimental era de 19, se dividió este número de modo equitativo, dando como resultado los tres subgrupos finales. Concluidas las sesiones de entrenamiento, se aplicó de nuevo el CDI y el CASQ-T a todos los participantes. En la tercera fase, se entrenó al grupo experimental de acuerdo con lo descrito en 
el componente de Habilidades de Comunicación. Se realizó en las mismas condiciones que la segunda fase. Concluidas las sesiones de entrenamiento, se aplicaron el CDI, el CASQ-T, el CEDIA y la ESV a todos los participantes. La fase de seguimiento se realizó seis meses después de la última sesión del componente de Habilidades de Comunicación. El CDI, el CASQ-T, el CEDIA y la ESV fueron administrados de nuevo a los 38 participantes en las mismas condiciones descritas.

Tanto la evaluación como la aplicación del entrenamiento fueron realizadas por el primer autor, psicólogo sanitario con experiencia en la intervención psicológica con adolescentes.

\section{Programa de Intervención}

El programa utilizado con el grupo tratado constó de 5 sesiones de tratamiento en grupo, de 2 horas de duración por sesión y periodicidad semanal. Los componentes del programa han sido tres: a) Educativo (1 sesión). Se expusieron las condiciones del entrenamiento y cuáles iban a ser los contenidos principales y se realizó la nivelación de expectativas. Se explicó en qué consiste el autorregistro y sus ventajas, así como el papel que iban a desempeñar las tareas para casa en el tratamiento. b) Entrenamiento para fomentar el estilo explicativo-atributivo optimista (2 sesiones). Se proporcionó una breve explicación de la terapia cognitiva, del pensamiento optimista y de sus efectos a distintos niveles. Se identificaron los pensamientos negativos/pesimistas y las consecuencias que tienen en el ámbito conductual y emocional, y se enseñó a sustituir esos pensamientos por otros más positivos/optimistas y a detectar las nuevas consecuencias conductuales y emocionales. Se corrigieron los autorregistros de los participantes, tomando como ejemplos al menos 3 de cada alumno, y dejando al resto de participantes que diesen su opinión con respecto a los pensamientos negativos, sus consecuencias y los posibles pensamientos positivos alternativos con sus consecuencias. c) Entrenamiento en Habilidades de Comunicación (2 sesiones). Se explicó qué son las habilidades interpersonales, cuáles son sus características, las ventajas de poseerlas y los inconvenientes de carecer de ellas. También se diferenció entre la conducta verbal y no verbal y los tres estilos de comunicación que hay (pasivo, agresivo y asertivo), indicándose cuáles son sus consecuencias en la comunicación con los demás. Se analizaron las tareas mandadas para casa y se facilitó que todos los participantes del grupo comentasen sus ejemplos. Posteriormente, se enseñaron habilidades para iniciar y mantener conversaciones, pedir citas y dar y recibir cumplidos. Por último, se pidió a los y las participantes que practicaran todo lo aprendido con amigos y compañeros.

\section{Análisis estadístico}

Para evaluar los efectos generales del tratamiento sobre el estilo explicativo y la sintomatología depresiva, se llevó a cabo un Análisis de Varianza Multivariado (MANOVA), con dos factores intergrupo: sexo (hombre y mujer) y grupo (experimental y control), y un factor de medidas repetidas: la fase del entrenamiento (pre, post 3 sesiones, post 5 sesiones y seguimiento). Por su parte, para determinar los efectos generales del entrenamiento sobre las habilidades de interacción con otros adolescentes y la satisfacción con la vida, se llevó a cabo un Análisis de Varianza Multivariado (MANOVA), con dos factores intergrupo: sexo (hombre y mujer) y grupo (experimental y control), y un factor de medidas repetidas: fase del entrenamiento (pre, post 5 sesiones y seguimiento). Cuando no se cumplió el supuesto de esfericidad en los análisis de medidas repetidas, se utilizaron los valores de la $\mathrm{F}$ corregidos mediante la prueba de Greenhouse-Geisser.

En todos los casos, los efectos intergrupo se contrastaron mediante ANOVAs univariados de un factor. Por su parte, las comparaciones para muestras relacionadas se hicieron a partir del estadístico t de Student. Por último, cuando no se cumplían los supuestos para la aplicación de pruebas paramétricas en los contrastes intergrupo, se usó la U de Mann Wittney.

Para los contrastes intergrupo, el tamaño del efecto se calculó mediante el índice de $\varepsilon^{2}$ parcial. Para calcular el tamaño del efecto teniendo en cuenta grupo y fase del entrenamiento, se usó el índice $d$, siendo éste la diferencia de cambios medios tipificada (incluyendo desviaciones típicas y tamaño muestral), resultante de comparar las diferencias entre pretest-postest 3 sesiones, pretest-postest 5 sesiones y pretest-seguimiento del grupo tratado menos las del grupo control. Para ello se usó la ecuación de Morris (2008), pero puede comprobarse una descripción más detallada de este procedimiento en la página 9 del trabajo de Sánchez-Meca, Marín-Martínez y LópezLópez (2011). Este segundo índice es complementario al primero y se usa fundamentalmente en estudios en los que quiere comprobarse la eficacia de tratamientos clínicos cuando se usan diseños complejos. De acuerdo con él, se habla de tamaño bajo, medio y alto con valores en torno a .20, .50 y .80, respectivamente (Cohen, 1988).

Aunque no se presentaban hipótesis específicas sobre la variable sexo, se ha incluido su análisis tanto de modo individual como a través de las interacciones con otras variables independientes. 
Para la realización de todos los análisis mencionados se utilizó el paquete estadístico SPSS 17.0.

\section{Resultados}

\section{Efectos del programa de entrenamiento relacionados con el sexo de los participantes}

No se encontró ningún efecto significativo de Sexo ni de Sexo x Fase del Entrenamiento en ninguna de las variables dependientes del estudio.

\section{Efectos del programa de entrenamiento sobre el estilo explicativo}

Los resultados del MANOVA 2x2 (x4) mostraron efectos significativos para la interacción Grupo x Fase del Entrenamiento $\left(\mathrm{F}_{(3,34)}=6.64 ; \mathrm{MC}=89.28 ; p<.001\right.$; $\varepsilon^{2}=.163$; s.p. $($ potencia $\left.)=.97\right)$.

Para determinar si existían diferencias entre los grupos experimental y control en las distintas fases del entrenamiento, se llevó a cabo un ANOVA de un factor (Grupo). Los resultados mostraron que no existían diferencias significativas en el estilo explicativo entre ambos grupos en la fase pretratamiento $\left(F_{(1,34)}=1.21 ; \mathrm{MC}=28.36 ; p=.279 ; \mathcal{E}^{2}=.034 ;\right.$ s.p. $\left.=.18\right)$, ni tras el seguimiento $\left(F_{(1,34)}=1.28 ; \mathrm{MC}=24.12 ; p=\right.$ $.264 ; \varepsilon^{2}=.036 ;$ s.p. $=.20$ ). Sin embargo, se hallaron diferencias marginalmente significativas después de las tres primeras sesiones de entrenamiento $(U=121.5 ; p=.08)$ y diferencias significativas después de las cinco sesiones de entrenamiento $\left(F_{(1,34)}=20.32 ; \mathrm{MC}=310.51 ; p\right.$ $<.001 ; \varepsilon^{2}=.374 ;$ s.p. $\left.=.99\right)$. En ambos casos, el estilo explicativo era más optimista en el grupo experimental (ver Tabla 1).

\section{Tabla 1}

Medias y desviaciones típicas (entre paréntesis) de las variables dependientes del estudio durante las distintas fases del entrenamiento

\begin{tabular}{|c|c|c|c|c|c|c|}
\hline \multirow[t]{2}{*}{ Variables } & \multirow[t]{2}{*}{ Fase entrenamiento } & \multicolumn{2}{|c|}{ Grupo Experimental } & \multicolumn{2}{|c|}{ Grupo Control } & \multirow[t]{2}{*}{$\mathrm{TE}(d)$} \\
\hline & & $\mathrm{N}$ & M (DT) & $\mathrm{n}$ & M (DT) & \\
\hline \multirow[t]{4}{*}{ Estilo explicativo } & Pretest & 19 & $1.36(4.29)$ & 19 & $3.00(5.12)$ & \\
\hline & Post. 3 sesiones & 19 & $5.31(3.88)$ & 19 & $3.00(4.35)$ & \\
\hline & Post. 5 sesiones & 19 & $8.00(4.29)$ & 19 & $2.42(3.71)$ & \\
\hline & Seguimiento & 19 & $2.21(5.05)$ & 19 & $0.57(3.74)$ & \\
\hline \multirow[t]{4}{*}{ Síntomas depresivos } & Pretest & 19 & $17.89(2.51)$ & 19 & $16.78(2.39)$ & \\
\hline & Post. 3 sesiones & 19 & $7.52(3.51)$ & 19 & $16.63(3.77)$ & 4.08 \\
\hline & Post. 5 sesiones & 19 & $5.47(3.28)$ & 19 & $16.15(3.37)$ & 4.71 \\
\hline & Seguimiento & 19 & $4.89(2.33)$ & 19 & $15.42(3.15)$ & 4.65 \\
\hline \multirow{4}{*}{$\begin{array}{l}\text { Dificultades para } \\
\text { las relaciones } \\
\text { heterosexuales }\end{array}$} & Pretest & 19 & $11.94(8.01)$ & 19 & $11.94(8.40)$ & \\
\hline & Post. 3 sesiones & & & & & \\
\hline & Post. 5 sesiones & 19 & $5.47(4.67)$ & 19 & $9.94(5.10)$ & 0.53 \\
\hline & Seguimiento & 19 & $4.94(4.08)$ & 19 & $8.10(4.29)$ & 0.38 \\
\hline \multirow[t]{3}{*}{$\begin{array}{l}\text { Dificultades para las } \\
\text { relaciones con amigos }\end{array}$} & $\begin{array}{l}\text { Pretest } \\
\text { Post. } 3 \text { sesiones }\end{array}$ & 19 & $1.94(2.22)$ & 19 & $3.10(3.88)$ & \\
\hline & Post. 5 sesiones & 19 & $1.26(2.37)$ & 19 & $3.63(2.71)$ & 0.05 \\
\hline & Seguimiento & 19 & $1.52(1.80)$ & 19 & $3.94(2.77)$ & -0.14 \\
\hline \multirow[t]{4}{*}{ Satisfacción vital } & Pretest & 19 & $16.31(4.84)$ & 19 & $16.73(6.09)$ & \\
\hline & Post. 3 sesiones & & & & & \\
\hline & Post. 5 sesiones & 19 & $23.15(4.36)$ & 19 & $15.63(4.38)$ & -1.02 \\
\hline & Seguimiento & 19 & $23.73(4.29)$ & 19 & $15.63(4.38)$ & -1.12 \\
\hline
\end{tabular}


Cuando se analiza por separado cada uno de los grupos, se observa un efecto significativo para el Factor Fase del Entrenamiento en el grupo experimental $\left(F_{(3,17)}\right.$ $=14.28 ; \mathrm{MC}=175.33 p<.001 ; \mathcal{E}^{2}=.457 ;$ s.p. $\left.=1\right)$, pero no en el grupo control $\left(F_{(2.24,17)}=1.90 ; \mathrm{MC}=37.16 ; p\right.$ $=.158 ; \varepsilon^{2}=.101 ;$ s.p. $\left.=.39\right)$. Las comparaciones con el estadístico t para muestras relacionadas realizadas en el grupo experimental muestran diferencias significativas en el estilo explicativo entre la fase pre-tratamiento y la medida realizada después de las tres primeras sesiones del tratamiento $\left(t_{(18)}=-3.87 ; p<.001\right)$, entre la fase pretratamiento y la medida realizada después de las cinco sesiones que constituyen el tratamiento $\left(t_{(18)}=-5.63 ; p\right.$ $<.001)$ y entre la finalización del tratamiento completo y el período de seguimiento $\left(t_{(18)}=5.20 ; p<.001\right)$. Los resultados indican que el entrenamiento realizado con el grupo experimental desarrolló un estilo explicativo más optimista tras las tres primeras sesiones y tras las cinco sesiones completas; sin embargo, el estilo explicativo optimista no se mantuvo durante el seguimiento.

\section{Efectos del programa de entrenamiento sobre la sintomatología depresiva}

Los resultados del MANOVA 2x2 (x4) mostraron efectos significativos para la interacción Grupo $\mathrm{x}$ Fase del Entrenamiento $\left(\mathrm{F}_{(2.1134)}=52.92 ; \mathrm{MC}=424.77 ; p\right.$ $<.001 ; \varepsilon^{2}=.609 ;$ s.p. $=1$ ), con tamaños del efecto $\mathrm{y}$ potencia altos.

Para determinar si existían diferencias entre los grupos experimental y control en las distintas fases del entrenamiento, se llevó a cabo un ANOVA de un factor (Grupo). Los resultados mostraron que no existían diferencias significativas en la sintomatología depresiva entre ambos grupos en la fase pretratamiento $\left(F_{(1,34)}=1.68 ; \mathrm{MC}=10.34 ; p=.202 ; \mathcal{E}^{2}=.047 ;\right.$ s.p. $\left.=.24\right)$, pero sí después de las tres primeras sesiones de entrenamiento $\left(F_{(1,34)}=62.57 ; \mathrm{MC}=803.32 ; p<.001 ; \varepsilon^{2}=\right.$ .648 ; s.p. $=1)$, después de las cinco sesiones de entrenamiento $\left(F_{(1,34)}=94.39 ; \mathrm{MC}=1086.37 ; p<.001 ; \varepsilon^{2}\right.$ $=.735$; s.p. $=1)$ y tras el seguimiento $\left(F_{(1,34)}=131.47\right.$; $\mathrm{MC}=1035.68 ; p<.001 ; \varepsilon^{2}=.795 ;$ s.p. $\left.=1\right)$, siendo el nivel de síntomas depresivos más bajo en el grupo experimental que en el grupo control en todos los casos. Los tamaños del efecto fueron grandes tras las tres sesiones de entrenamiento, tras las cinco sesiones de entrenamiento y en la fase de seguimiento (ver Tabla 1).

Por último, cuando se analiza por separado cada uno de los grupos, se observa un efecto significativo para el Factor Fase del Entrenamiento en el grupo experimental $\left(F_{(1.74,29.60)}=104.07 ; \mathrm{MC}=1186.69 p\right.$
$<.001 ; \varepsilon^{2}=.860 ;$ s.p. $=1$ ), pero no en el grupo control $\left(F_{(3,51)}=1.66 ; \mathrm{MC}=7.81 ; p=.187 ; \varepsilon^{2}=.089 ;\right.$ s.p.=.41 $)$. Las comparaciones con el estadístico t para muestras relacionadas realizadas en el grupo experimental muestran diferencias significativas en la sintomatología depresiva entre la fase pre-tratamiento y la medida realizada después de las tres primeras sesiones del tratamiento $\left.t_{(18)}=9.70 ; p<.001\right)$ y entre la fase pre-tratamiento y la medida realizada después de las cinco sesiones que constituyen el tratamiento $\left(t_{(18)}=11.68 ; p<.001\right)$, siendo el nivel de síntomas depresivos más bajo después de las tres primeras sesiones de entrenamiento y después de las cinco sesiones del tratamiento, respectivamente. Finalmente, se observó que la media de síntomas depresivos obtenida tras el seguimiento no difería de la media de síntomas depresivos obtenida después de las cinco sesiones de tratamiento $\left(t_{(18)}=0.97 ; p=.343\right)$.

\section{Efectos del programa de entrenamiento sobre las habilidades de interacción con otros adolescentes}

\section{Escala de Relaciones Heterosexuales}

El MANOVA 2x2 (x3) no mostró efectos significativos para ninguna de las interacciones.

Para determinar si existían diferencias entre los grupos experimental y control en las distintas fases del entrenamiento, se llevó a cabo un ANOVA de un factor (Grupo). Los resultados mostraron que no existían diferencias significativas en la puntuación de dificultades heterosexuales entre ambos grupos en la fase pretratamiento $\left(F_{(1,34)}=0.46 ; \mathrm{MC}=3.11 ; p=.832 ; \varepsilon^{2}=.001\right.$; s.p. $=.05)$, pero sí existían diferencias significativas entre ambos grupos después de las cinco sesiones de entrenamiento $\left(F_{(1,34)}=6.47 ; \mathrm{MC}=148.21 ; p<.016 ; \varepsilon^{2}\right.$ $=.160 ;$ s.p. $=.69) \mathrm{y}$ tras el seguimiento $\left(F_{(1,34)}=4.42\right.$; $\mathrm{MC}=77.78 ; p<.04 ; \varepsilon^{2}=.115 ;$ s.p. $\left.=.53\right)$, siendo la puntuación de dificultades heterosexuales menor en el grupo experimental que en el grupo control. El tamaño del efecto fue medio tras las cinco sesiones de entrenamiento y bajo en la fase de seguimiento (ver Tabla 1).

Finalmente, cuando se analiza por separado cada uno de los grupos, se observa un efecto significativo para el Factor Fase del Entrenamiento en el grupo experimental $\left(F_{\left(1^{\prime} 49,26^{\prime} 93\right)}=19.79 ; \mathrm{MC}=385.88 ; p<.001\right.$; $\varepsilon^{2}=.524 ;$ s.p. $=.99$ ), pero no en el grupo control $\left(F_{\left(1448.26{ }^{\prime} 6\right)}=2.92 ; \mathrm{MC}=94.72 ; p=.084 ; \varepsilon^{2}=.14 ;\right.$ s.p. $\left.=.45\right)$. Las comparaciones con el estadístico t para muestras 
relacionadas realizadas en el grupo experimental muestran diferencias significativas en el nivel de dificultades heterosexuales entre la fase pre-tratamiento y la medida realizada después de las cinco sesiones que constituyen el tratamiento $\left(t_{(18)}=4.83 ; p<.001\right)$, siendo el nivel medio de dificultades heterosexuales menor después de las cinco sesiones del tratamiento. Finalmente, se observó que la media de dificultades heterosexuales obtenida tras el seguimiento no difería de la media obtenida después de las cinco sesiones de tratamiento $\left(t_{(18)}=0.64 ; p=.528\right)$.

\section{Escala de Relaciones con Amigos}

El MANOVA 2x2 (x3) no mostró efectos significativos para ninguna de las interacciones.

Para determinar si existían diferencias entre los grupos experimental y control en las distintas fases del entrenamiento, se llevó a cabo un ANOVA de un factor (Grupo). Los resultados mostraron que no existían diferencias significativas en la puntuación de dificultades con los amigos entre ambos grupos en la fase pretratamiento $(U=159.5 ; p=.54)$, pero sí existían diferencias significativas entre ambos grupos después de las cinco sesiones de entrenamiento $\left(F_{(1.34)}=8.56\right.$; $\mathrm{MC}=57.37 ; p<.006 ; \varepsilon^{2}=.201 ;$ s.p. $\left.=.81\right)$ y tras el seguimiento $(U=80.00 ; p<.003)$, siendo la puntuación de dificultades con los amigos menor en el grupo experimental que en el grupo control. No obstante, el tamaño del efecto fue prácticamente nulo tras las cinco sesiones de entrenamiento y tras la fase de seguimiento (ver Tabla 1).

Finalmente, cuando se analiza por separado cada uno de los grupos, no se observa ningún efecto significativo para el Factor Fase del Entrenamiento en el grupo experimental $(W=.04 ; p=.40)$, ni en el grupo de control $\left(F_{(1.46,24.96)}=0.88 ; \mathrm{MC}=6.14 ; p=.395 ; \varepsilon^{2}\right.$ $=.050 ;$ s.p. $=.16)$.

\section{Efectos del programa de entrenamiento sobre la satisfacción vital}

Los resultados del MANOVA 2x2 (x3) mostraron efectos significativos para la interacción Grupo x Fase del Entrenamiento $\left(F_{(1.68,57.10)}=25.21 ; \mathrm{MC}=274.48 ; p\right.$ $<.001 ; \varepsilon^{2}=.426 ;$ s.p. $=1$ ), siendo el tamaño del efecto medio y la potencia alta.

Para determinar si existían diferencias entre los grupos experimental y control en las distintas fases del entrenamiento, se llevó a cabo un ANOVA de un factor (Grupo). Los resultados mostraron que no existían diferencias significativas en los niveles de satisfacción vital entre ambos grupos en la fase pretratamiento $\left(F_{(1,34)}=0.10 ; \mathrm{MC}=3.36 ; p<.747 ; \mathcal{E}^{2}=.003 ;\right.$ s.p. $\left.=.06\right)$, pero sí después de las cinco sesiones de entrenamiento $\left(F_{(1,34)}=29.24 ; \mathrm{MC}=555.55 ; p<.001 ; \varepsilon^{2}=.462 ;\right.$ s.p. $\left.=.99\right)$ y durante el seguimiento $\left(F_{(1,34)}=34.20 ; \mathrm{MC}=637.40\right.$; $p<.001 ; \varepsilon^{2}=.501 ;$ s.p. $=1$ ), siendo los niveles de satisfacción vital mayores en el grupo experimental que en el grupo control. Los tamaños del efecto fueron grandes tras las cinco sesiones de entrenamiento y en la fase de seguimiento (ver Tabla 1).

Cuando se analiza por separado cada uno de los grupos, se observa un efecto significativo para el Factor Fase del Entrenamiento en el grupo experimental $\left(F_{(2.36)}=35.10 ; \mathrm{MC}=323.70 ; p<.001 ; \mathcal{E}^{2}=.661 ;\right.$ s.p. $\left.=1\right)$, pero no en el grupo control $\left(F_{(2,36)}=0.82 ; \mathrm{MC}=7.73\right.$; $p<.44 ; \varepsilon^{2}=.04$; s.p. $=.18$ ). Las comparaciones con el estadístico t para muestras relacionadas realizadas en el grupo experimental muestran diferencias significativas en las puntuaciones de satisfacción vital entre la fase pre-tratamiento y la medida realizada después de las cinco sesiones que constituyen el tratamiento $\left.t_{(18)}=-7.80 ; p<.001\right)$, siendo los niveles de satisfacción vital mayores después de haber recibido las cinco sesiones del tratamiento. Finalmente, se observó que los niveles de satisfacción vital obtenidos tras el seguimiento no diferían de los niveles obtenidos después de las cinco sesiones de tratamiento $\left(t_{(18)}=-0.56 ; p=.579\right)$.

\section{Discusión}

El objetivo de este estudio fue comprobar la eficacia de un programa de entrenamiento para adolescentes, centrado en el desarrollo de un estilo explicativo optimista, la disminución de la sintomatología depresiva y el incremento de la satisfacción con la vida y las habilidades de interacción con otros adolescentes. El programa de entrenamiento ha demostrado tener efectos positivos significativos sobre todas las variables evaluadas.

En principio, se esperaba que el programa produjera un cambio hacia un estilo explicativo más optimista en el grupo de adolescentes que recibió un entrenamiento para fomentarlo. El análisis de los datos indica que el entrenamiento recibido fue eficaz para incrementar este estilo explicativo, sobre todo con la combinación de los componentes cognitivo e interpersonal. Estos datos confirman lo obtenido en otros estudios, con distintos programas de entrenamiento (Brunwasser et al., 2009; Gillham et al., 2001; Miloseva, 2012; Shatte et al., 2000), 
y corrobora la validez del procedimiento empleado para lograr el objetivo propuesto. Sin embargo, los efectos obtenidos no perduraron tras el seguimiento realizado, a diferencia de lo sucedido en los estudios mencionados, pero igual que sucede en otros estudios (Gilboy, 2003). Podría ser que el tratamiento utilizado en nuestro caso no sea suficientemente potente para generar un estilo explicativo más optimista a largo plazo; así, la falta de práctica posterior con el procedimiento aprendido ha podido favorecer el olvido y el mantenimiento del aprendizaje o puede ser que los y las estudiantes hayan tenido dificultad para traducir lo aprendido en comportamientos concretos que formen parte de su estilo de comportamiento de modo relativamente permanente. También es posible que la prueba de evaluación empleada para medir el estilo explicativo resulte excesivamente complicada de entender por parte de los adolescentes. Por último, podría deberse igualmente a las dificultades que se han producido para recoger los datos del seguimiento (en un curso académico distinto a aquél en el que se realizó el tratamiento y con problemas para reunir a todos los participantes). De cualquier modo, será necesario realizar estudios posteriores que nos permitan comprobar cada una de estas posibilidades.

Además, se pretendía que el programa de entrenamiento redujera los síntomas depresivos e incrementara la satisfacción vital y las habilidades de interacción interpersonal en los adolescentes del grupo experimental. Los datos recogidos permiten afirmar que el programa de entrenamiento aplicado logra los cambios esperados en todas las variables, y que éstos se mantienen tras el seguimiento. Los cambios fueron de gran magnitud en general, como ha puesto de manifiesto el análisis de los tamaños del efecto. Estos resultados confirman los datos existentes en la literatura sobre los beneficios de aplicar programas de entrenamiento centrados en el estilo explicativo optimista (solo o en combinación con el entrenamiento en habilidades interpersonales) para reducir los síntomas depresivos en adolescentes (Brunwasser et al., 2009; Cantrell, 2006; Gillham et al., 2001; Miloseva, 2012; Shatte et al., 2000; Wijnhoven et al., 2014) y para incrementar la satisfacción vital o la felicidad (Cantrell, 2006; Demir \& Weitekamp, 2007; Suldo et al., 2014). En el adolescente, los síntomas depresivos pueden estar relacionados con sus atribuciones con respecto a los acontecimientos negativos que le suceden, pudiendo estas atribuciones, a su vez, influir en las expectativas y la motivación futuras que desarrolla para enfrentarse con distintas situaciones de tipo interpersonal. Si esto se une con dificultades comportamentales concretas para desenvolverse con otros adolescentes de modo exitoso, es probable que disminuya también el contacto social y la adaptación durante este período, lo que podría aumentar el nivel de sintomatología depresiva y reducir la satisfacción vital, estableciéndose un círculo cognitivo-comportamental cuyas consecuencias negativas se convierten en algo estable. Por ello, la aplicación de programas de intervención como el que se ha administrado, en los que se combinan, por un lado, el entrenamiento en valoraciones positivas de los acontecimientos y de la propia persona (con el subsecuente cambio cognitivo) y, por otro lado, el entrenamiento en habilidades sociales, producen efectos muy positivos en el adolescente a todos los niveles y se convierten en una herramienta poderosa para mejorar su salud, su adaptación y su satisfacción vital.

Aunque los resultados de este estudio son sin duda prometedores, éste presenta algunas limitaciones que habrán de tenerse en cuenta y subsanarse mediante trabajos posteriores. La primera de ellas está relacionada con el tamaño de la muestra utilizada: ésta es reducida y la distribución por sexos no ha sido homogénea entre ambos grupos experimentales, lo que limita la posibilidad de generalización de los resultados encontrados. La segunda, concierne a la duración del entrenamiento aplicado y los grupos de control utilizados en el estudio. A pesar de que el reducido número de sesiones es una ventaja con respecto a otro tipo de intervenciones igualmente eficaces (pero más costosas en términos de tiempo) habría que hacer una comparación entre ellas para comprobar este aspecto, diseñando un estudio que tenga en cuenta otros tipos de tratamiento centrados en el entrenamiento del pensamiento positivo. Por otra parte, también podría ser interesante estudiar si este tipo de intervenciones puede mejorar el rendimiento de los/as alumnos/as, actuando sobre él indirectamente y, en este sentido, tendría que compararse igualmente con las intervenciones que influyen favorablemente en esta variable. La última limitación se debe al procedimiento seguido para recoger los datos. $\mathrm{Al}$ tener que realizarse la evaluación en un centro educativo, y estar sometida a las propias reglas del centro y a los grupos a los que pertenecen los alumnos, es conveniente realizar la totalidad del estudio en un mismo curso académico, hecho que no se ha producido en esta ocasión en cuanto a la evaluación de las variables en el seguimiento. Es posible que esto haya podido influir de algún modo en los resultados obtenidos. Por tanto, en estudios posteriores, sería recomendable comenzar con el programa de entrenamiento a principios del curso académico y terminar con el seguimiento antes de finalizar éste. 


\section{Referencias}

Alloy, L., Abramson, L., Whitehouse, W., Hogan, M., Panzarella, C. \& Rose, D. (2006). Prospective incidence of first onsets and recurrences of depression in individuals at high and low cognitive risk for depression. Journal of Abnormal Psychology, 115(1), 145-156. doi:10.1037/0021-843X.115.1.145

Berger, B., Owen, D. \& Man, F. (1993). A brief review of literature and examination of acute mood benefits of exercises in Czechoslovakian and United States swimmers. International Journal of Sport Psychology, 24(2), 130-150. Recuperado de http://www. cabdirect.org/abstracts/19931860420.html

Brunwasser, S., Gillham, J. \& Kim, E. (2009). A meta-analytic review of the Penn Resiliency Program. Journal of Consulting and Clinical Psychology, 77(6), 1042-1054. doi:10.1037/a0017671

Cantrell, K. (2006). An exploratory examination of the effectiveness of explanatory style for positive versus negative events in group cognitive behavioral treatment. Tesis Doctoral no publicada. Laramie, Wyoming: University of Wyoming.

Cardemil, E., Reivich, K. \& Seligman, M. (2002). The prevention of depressive symptoms in low-income minority middle school students. Behaviour Research and Therapy, 5(2), 313-327. doi:10.1037/1522-3736.5.1.58a

Chan, S. (2012). Early adolescent depressive mood: Direct and indirect effects of attributional styles and coping. Child Psychiatry \& Human Development, 43(3), 455-470. doi:10.1007/ s10578-011-0275-9

Chaplin, T., Gillham, J., Reivich, K., Elkon, A., Samuels, B., Freres, D., Winder, B. \& Seligman, M. (2006). Depression prevention for early adolescent girls. A pilot study of all girls versus coed groups. The Journal of Early Adolescence, 26(1), 110-126. Doi: $10.1177 / 0272431605282655$

Cohen, J. (1988). Statistical power analysis for the behavioral sciences. Hillsdale: Erlbaum.

Del Barrio, V. \& Carrasco, M. (2004). CDI. Inventario de Depresión Infantil. Madrid: TEA ediciones.

Demir, M. \& Weitekamp, L. (2007). I am so happy cause today I found my friend: Friendship and personality as predictors of happiness. Journal of Happiness Studies, 8(2), 181-211. doi:10.1007/ s10902-006-9012-7

Diener, E., Emmons, R., Larsen, R. \& Griffin, S. (1985). The Satisfaction with Life Scale. Journal of Personality Assessment, 49(1), 71-75. doi:10.1207/s15327752jpa4901_13

Frydenberg, E. (1997). Adolescent Coping. Londres: Routledge.

Garber, J. (2000). Optimism: Definitions and origins. En J.E. Gillham (Ed.). The science of optimism and hope (pp. 299-314). Philadelphia: Templeton Foundation.

Gibb, B. \& Alloy, L. (2006). A prospective test of the hopelessness theory of depression in children. Journal of Clinical Child \& Adolescent Psychology, 35(2), 264-274. doi:10.1207/ s15374424jccp3502_10

Gilboy, S. (2003). Students'optimistic attitudes and resiliency program. Empirical validation of a prevention program developing hope and optimism. Tesis Doctoral no publicada. Arizona: University of Arizona.

Gillham J., Brunwasser S.M. \& Freres D.R. (2008). Preventing depression in early adolescence. En J.R.Z. Abela \& B.L. Hankin (Eds.). Handbook of depression in children and adolescents (pp. 309-322). New York: Guilford Press.

Gillham, J., Hamilton, J., Freres, D., Patton, K. \& Gallop, R. (2006). Preventing depression among early adolescents in the primary care setting: a randomized controlled study of the Penn Resiliency Program. Journal of Abnormal Child Psychology, 34(2), 203-219. doi:10.1007/s10802-005-9014-7
Gillham, J. \& Reivich, K. (2004). Cultivating optimism in childhood and adolescence. Annals of the American Academy of Political and Social Science, 591(1), 146-163. doi:10.1177/0002716203260095 Gillham, J., Reivich, K., Jaycox, L. \& Seligman, M. (1995). Prevention of depressive symptoms in schoolchildren: Two year follow up. Psychological Science, 6(6), 343-351. Recuperado de http:// www.jstor.org/stable/40062887

Gillham, J., Shatte, A., Reivich, K. \& Seligman, M. (2001). Optimism, pessimism, and explanatory style. En E.C. Chang (Ed.). Optimism \& pessimism: Implications for theory, research, and practice (pp. 53-75). Washington D.C.: American Psychological Association.

Griciute, A. (2011). Analysis of association between depressive symptoms and resilience in secondary school student groups. International Journal of Psychology: A Biopsychosocial Approach, 9, 9-28. Recuperado de http://www.psyjournal.vdu.lt/wp/2011no-9/analysis-of-association-between-depressive-symptoms-andresilience-in-secondary-school-student-groups/

Hankin, B. (2008). Cognitive vulnerability-stress model of depression during adolescence: Investigating depressive symptom specificity in a multi-wave prospective study. Journal of Abnormal Child Psychology, 36(7), 999-1014. doi:10.1007/s10802-008-9228-6

Hankin, B. \& Abramson, L. (2001). Development of gender differences in depression: An elaborated cognitive vulnerability-transactional stress theory. Psychological Bulletin, 127(6), 773-796. doi:10.1037/0033-2909.127.6.773

Inglés, C., Méndez, F., Hidalgo, M., Rosa, A. \& Orgilés, M. (2003). Cuestionarios, inventarios y escalas de ansiedad social para adolescentes: Una revisión crítica. Revista de Psicopatología y Psicología Clínica, 8(1), 1-21. doi:10.5944/rppc.vol.8.num.1.2003.3939

Inglés Saura, C., Méndez, F. \& Hidalgo, M. (2000). Cuestionario de Evaluación de Dificultades Interpersonales en la Adolescencia. Psicothema, 12(3), 390-398. Recuperado de http://www.psicothema.com/psicothema. $\cdot$ sp $? \mathrm{id}=347$

Jacobs, R., Reinecke, M., Gollan, J. \& Kane, P. (2008). Empirical evidence of cognitive vulnerability for depression among children and adolescents: A cognitive science and developmental perspective. Clinical Psychology Review, 28(5), 759-782. doi:10.1016/j. cpr.2007.10.006

Jaycox L., Reivich K., Gillham J. \& Seligman, M. (1994). Prevention of depressive symptoms in school children. Behaviour Research and Therapy, 32(8), 801-816. doi:10.1016/0005-7967(94)90160-0

Kindt, K., Van Zundert, R. \& Engels, R. (2012). Evaluation of a Dutch school-based depression prevention program for youths in highrisk neighborhoods: study protocol of a two-armed randomized controlled trial. Biomedical Central Public Health, 12, 212-218. doi:10.1186/1471-2458-12-212

Know, P. \& Laurenceau, J. (2002). A longitudinal study of the hopelessness theory of depression: Testing the diathesis-stress model within a differential reactivity exposure framework. Journal of Clinical Psychology, 58(10), 1305-1321. doi:10.1002/jclp.10043

Kovacs, M. (1992). Children's depression inventory: manual. North Tonawanda, NY: Multi-Health Systems.

Lewinsohn, P., Pettit, J., Joiner, T. \& Seeley, J. (2003). The symptomatic expression of major depressive disorder in adolescents and young adults. Journal of Abnormal Psychology, 112(2), 244-253. doi: $10.1037 / 0021-843 X .112 .2 .244$

López Rodríguez, E. (2003). Propiedades psicométricas de una versión española del Cuestionario de Estilo Atribucional (OptimistaPesimista) para niños (CASQ-T) en una muestra de la provincia de Jaén. (Tesis de maestría inédita), Jaén, España.

Miloseva, L. (2012). A cognitive behavioral depression prevention program for early adolescents. Journal on Educational Psychology, 6(3), 32-40. 
Mongrain, M. \& Blackburn, S. (2005). Cognitive vulnerability, lifetime risk, and the recurrence of major depression in graduate students. Cognitive Therapy and Research, 29(6), 747-768. doi:10.1007/s10608-005-4290

Morris, S. (2008). Estimating effects sizes from pretest-posttestcontrol group designs. Organizational Research Methods, 11(2), 364-386. doi:10.1177/1094428106291059

Nolen-Hoeksema, S., Girgus, J. \& Seligman, M. (1992). Predictors and consequences of childhood depressive symptoms: A 5-year longitudinal study. Journal of Abnormal Psychology, 101(3), 405-422. doi:10.1037/0021-843X.101.3.405

Nrughma, L., Holen, A. \& Sund, A. (2010). Associations between attempted suicide, violent life events, depressive symptoms, and resilience in adolescents and young adults. The Journal of Nervous and Mental Disease, 198(2), 131-136. doi:10.1097/ NMD.0b013e3181cc43a2

Rodríguez, E., Donnenberg, G., Emerson, E., Wilson, H. \& Javdani, S. (2015). Externalizing symptoms moderate associations among interpersonal skills, parenting, and depressive symptoms in adolescents seeking mental health treatment. Journal of Youth Adolescence, 44(4), 952-963. doi:10.1007/s10964-015-0263-7

Sánchez Hernández, O. \& Méndez Carrillo, F. (2009). El optimismo como factor protector de la depresión infantil y adolescente. Clínica y Salud, 20(3), 273-280. Recuperado de http://scielo.isciii. es/pdf/clinsa/v20n3/v20n3a08.pdf

Sánchez-Meca, J., Marín-Martínez, F. \& López-López, J. (2011). Meta-análisis e intervención psicosocial basada en la evidencia. Psychosocial Intervention, 20(1), 95-107. doi:10.5093/ in2011v20n1a8

Sanjuán, P. \& Magallares, A. (2006). Estilo atributivo negativo, sucesos vitales y sintomatología depresiva. Revista de Psicopatología y Psicología Clínica, 11(2), 91-98. doi:10.5944/rppc. vol.11.num.2.2006.4020

Sanjuán, P. \& Magallares, A. (2009). A longitudinal stydy of the negative explanatory style and attributions of uncontrollability as predictors of depressive symptoms. Personality and Individual Differences, 46(7), 714-718. doi:10.1016/j.paid.2009.01.030

Sanjuán, P., Pérez-García, A., Rueda, B. \& Ruiz, M. (2008). Estilos explicativos y afecto negativo. Revista de Psicopatología y Psicología Clínica, 13(1), 45-52. doi:10.5944/rppc.vol.13. num.1.2008.4049
Schwartz, A., Kaslow, N., Seeley, J. \& Lewinsohn, P. (2000). Psychological, cognitive, and interpersonal correlates of attributional change in adolescents. Journal of Clinical Child Psychology, 29(2), 188-198. doi:10.1207/S15374424jccp2902_5

Seligman, M., Peterson, C., Kaslow, N., Tanenbaum, R., Alloy, L. \& Abramson, L. (1984). Attributional style and depressive symptoms among children. Journal of Abnormal Psychology, 93(2), 235-238. doi:10.1037/0021-843X.93.2.235

Shatté, A.J., Gillham, J.E. \& Reivich, K. (2000). Promoting hope in children and adolescents. En J.E. Gillham (Ed.). The science of optimism and hope: Research essays in honor of Martin E. P. Seligman. Laws of life symposia series (pp. 215-234). West Conshohocken, PA: Templeton Foundation Press.

Southwick, S., Litz, B., Charney, D. \& Friedman, M. (2011). Resilience and mental health. Challenges across the lifespan. Cambridge: Cambridge University Press.

Sturman, E., Mongrain, M. \& Kohn, P. (2006). Attributional style as a predictor of hopelessness depression. Journal of Cognitive Psychotherapy, 20(4), 447-458. doi:10.1891/jcpiq-v20i4a008

Suldo, S., Savage, J. \& Mercer, S. (2014). Increasing Middle School Students' Life Satisfaction: Efficacy of a Positive Psychology Group Intervention. Journal of Happiness Studies, 15(1), 19-42. doi:10.1007/s10902-013-9414-2

Tak, Y., Van Zundert, R., Kuijpers, R., Van Vlokhoven, B., Rensink, H. \& Engels, R. (2012). A randomized controlled trial testing the effectiveness of a universal schoolbased depression prevention program 'Op Volle Kracht' in the Netherlands. Biomedical Central Public Health, 12, 21-29. doi:10.1186/1471-2458-12-21

Thompson, M., Kaslow, N., Weiss, B. \& Nolen-Hoeksema, S. (1998). Children's Attributional Style Questionnaire-Revised: Psychometric examination. Psychological Assessment, 10(2), 166-170. doi:10.1037/1040-3590.10.2.166.

Twenge, J. \& Nolen-Hoeksema, S. (2002). Age, gender, race, socioeconomic status, and birth cohort differences on the Children's Depression Inventory: A meta-analysis. Journal of Abnormal Psychology, 111(4), 578-588. doi:10.1037/0021-843X.111.4.578 Wijnhoven, L., Creemer, D., Vermulst, A., Scholte, R. \& Engels, R. (2014). Randomized controlled trial testing the effectiveness of a depression prevention program ('Op Volle Krtacht') among adolescent girls with elevated depressive symptoms. Journal of Abnormal Child Psychology, 42(2), 217-228. doi:10.1007/s10802-013-9773-5 